\title{
Psychotherapeutic Praxis in India: Incorporation and Application of Indigenous Worldviews
}

Rijul Jayaraj Ballal*

\begin{abstract}
This research aims to gain insight into the incorporation of indigenous worldviews on mental health. Unstructured interviews were used on 16 counsellors who practised in Bangalore following the phenomenological paradigm. The interviews were audiorecorded and transcribed following which it underwent the 4 stages in Giorgi's method. The revelatory themes obtained from this study were that clients viewed counsellors as authority figures, they experienced shame and guilt, lack of social support, ostracism, isolation, faced language barriers, feared diagnosis and medication and so on. The main conduit of religion and culture, the family, is influenced by class, education, rural or urban origin, joint or nuclear setup and so on. Counsellors developed the skills and sensitivity to respond to these demands.
\end{abstract}

Keywords: Counsellors, Indigenous Worldviews, Phenomenological.

\section{Introduction}

The Indian conception of the psyche constitutes a multitude of attitudes that originate in history, religion, gender or caste. How do counsellors accommodate both indigenous ideas and theoretical orientations that are not indigenous?

*Shikshakalp fellow, Ek Step; rijul.ballal@gmail.com 
Nandy(2004), describes scientific developments as plotted along two axes. The first consists of changes in the structure of scientific knowledge seen as valid, universal and cumulative. The second axis has the shifting concepts of science as a social activity, thought of as non-rational, biased and intrusive, though acknowledged as important. He locates a breakdown of this dichotomy as an opportunity to redefine the ethics of research in psychology.

No other science has had to grapple with this described "loss of innocence" about the nature of scientific knowledge more than psychology. With the need to examine the nature of the relationship between the therapist, client and their work articulated as early as Freud (King \& Brien, 2011), there exists a need to account for what Nandy describes as the plurality of indigenous psychologies. This is not restricted to accusations of fraudulent data sampling levelled against researchers like Cyril Burt, as Plucker (1997) describes, but as Gould (1981) argues, the doing away of traditional concepts of intellect, making intelligence an instrument that is biased to socio-economic status, and hegemonies the concept of intelligence to be what intelligence tests measure.

The Canadian psychologist Adair (1999) would define indigenous psychologies as "One in which theories, concepts, methods and measure emanate from and reflect back upon the culture in which the behaviour is studied".

From a theoretical standpoint, there have been attempts to articulate an Indian perspective on psychological dimensions such as Mishra's (2006) and Sinha's (1944). However, both these narratives exclude subaltern groups, removing Indian psychology from contemporary conditions. Kakar (1982) ingrained himself with local faith healers but does not account for counsellors as primary therapists.

In a study examining self-perceived limitations of psychotherapists in India, Bhola, Kumaria and Orlinsky (2012) noted that ignorance and stigma about counselling, poverty, illiteracy, religion and language created a great deal of variation. The professional development of counsellors was dependent on peer support and how organisations structured supervision (2017). Sriram and 
Duggal (2015) found that family counsellors in India have to acknowledge the changing socio-cultural realities of the Indian context with people seeking alternatives to their traditional practices.

Unlike existing material, this inquiry will go beyond the observation of phenomena that accompany indigenous worldviews or personal narratives of accommodation and will look at different counsellors to sample a variety of experiences and qualitative data that will comprehensively inform one about the nature of this process.

\section{Review of literature}

Nandy (2004) speculates about the implications of a variety of ideas from sources such as Nietzsche, Freud, the post-colonial and Madhyamika traditions. He focusses mainly on theory to support his arguments though he does offer real-world examples of how contradictions within the ideals or ethics of psychology may affect practice.

Similarly, Sinha (1944) does not explain much about what his extensive list of terms, ideas and concepts would mean in a therapeutic setting. The contradictory multi-religious conceptsoffer radically different ideas on the psyche. There are no attempts at reconciliation or an overarching model. The question of how applicable such an approach is when it excludes subaltern groups and developments in native psychologies is also pertinent.

Mishra (2006) actively compares historical Indian concepts to the constructionist perspective and builds a framework. He calls for more cross-cultural studies to explore these ideas but stops short of examining how theyarise within the therapeutic relationship. He also excludesperspectives like Sinha's.

Allwood (2002) distinguishes native psychological traditions from the indigenisation of psychology which he conceives of as a response to ideas and developments in the field of western psychology. Using Sinha's (1997) work to describe the sometimes subservient and ideological nature of Indian psychology, he 
elaborates the function of culture and normalisation in indigenous groups (Allwood, 2011).

Teo (2013) outlines trends in Filipino and German critical psychology as a response to a trend of Americanisation arguing that new developments in the various national traditions of psychology stem from an underlying desire of indigenous ideas to reassert themselves.

From a cultural psychology perspective, Shweder (2008)observes what it means to be sick in an Odishan village. Through conversation,language and translation, he reveals the radically different conceptions of personality, health and treatment in a universal experience.

In a similar approach Kakar (1982), also uses naturalistic observation placing himself in settings where indigenous healers address mental health. He engages both the clients and healers but does not interfere, noticing a lot of indirect allusions to psychoanalytical concepts. He describes "Indian" behaviour in psychosexual and psychosocial development (Kakar \& Kakar, 2007).

Arulmani (2007) describes Indian psychology as existing between two traditions Mano-Vidya embedded in the ancient guru-shishya tradition and the western tradition. He argues that Indian psychology has been dependent on western psychology, with poor conceptual clarity and organisational structures failing to address the unique needs of the Indian context such as social stratification, rural-urban migration and gender. He suggests developing a culturally relevant and dynamic model from both traditions, equipped to deal with Indian conditions.

Carson, Jain and Ramirez (2009) note that the family, the central influence within Indian society, is inadequately addressed in terms of the educational and training available to counsellors while the need for family counselling exists. They note that counsellingis poorly funded, supported by few institutions andthe idea that mental health issues are due to consumerism hinder the development of the mental health profession in India. 
In a detailed examination of the difficulties faced by Indian counsellors,Pereira and Rekha (2007) found issues such as inadequate practical training, insufficient pay, lack of cooperation of clients and an absence of a network or directory of other mental health professionals. The lack of licensing and a governing body meant that counsellor employers did not recognise their qualifications. Job security and pay were poor.

Common in these attempts at examining indigenous psychologies is a lack of evaluation of what the primary counsellor experiences and how these various psychologies affect the therapeutic process. There is a great amount of data that we may obtain from examining this unexplored area of research. This gap must be addressed if we are to call for more inclusive ethics in psychology.

\section{Method}

\subsection{Research Questions}

This research will seek to address 3 questions.

- What aspects of indigenous worldviews enter the therapeutic process?

- On what factors are they dependent and how do they influence the counselling process?

- How do counsellors accommodate these worldviews?

\subsection{Paradigm}

The research was from the perspective of Phenomenology, the study of the conscious experience from the first-person point of view. One may acquire a background of having lived through a given type of experience (Smith, 2018). In Phenomenology, research findings are analysed using concepts from phenomenological philosophy to understand the findings and enable better theoretical analysis (Davidsen, 2013).

Unstructured in-depth personal interviews were utilised. Harvey (2019) describes this method as one where the interviewer has no predetermined set of questions. The intervieweediscussesrelevant areas, the interviewer remains vague, responding to keep the interview going. 


\subsection{Setting}

Interviews were conducted face to face, in Bangalore at places convenient for the interviewees. Care was taken to ensure that the setting was one that was free of distraction with minimal interferences.

\subsection{Population and Participants}

16 participants were selected based on thepurposive sampling method. This research included mental health professionals of any age, qualification, and years of experience. Each person who agreed to participate was required to read and sign an informed consent form.

Table 1: Demography details of the participants

\begin{tabular}{lllll}
\hline Sample size & Male & Female & Age range & Mean age \\
\hline $\mathrm{N}=16$ & 2 & 14 & $23-58$ & 30 \\
\hline
\end{tabular}

More pertinent to this study was the fact that the counsellors interviewed included those who had worked with a wide range of people across religion, caste, socio-economic status and cultural backgrounds. They employed a range of different therapeutic models.

\subsection{Data Sources and Collection Process}

All interviews were audio-recorded and lasted around 30 minutes. Transcripts and observations from the interviews were made. The obtained data was analysed based on Giorgi's method.

\subsection{Data Coding}

The interviews were recorded and transcribed. The transcripts were evaluated and used to obtain information relevant to this study. Participants in the study have been coded as respondents such as R1, R2, R3 and so on.

\subsection{Data Analysis Strategy}

Whiting (2002) offers a method to analyse the data obtained through Phenomenology, based on Giorgi's method, which involves 4 major stages: phenomenological bracketing, identifying 
natural units, developing revelatory themes, and descriptive statements.

\subsection{Ethics}

The participants were informed about the purpose, procedure of the study, the confidentiality of the information shared and their right to withdraw from the study. Any doubts or questions raised by the participants were answered by the researcher and their consent was obtained.

\subsection{Findings}

Stage 1: The phenomenological bracketing includes the belief that religion influences the perception of clients, problems faced by clients were based on their socio-economic status, the family is the main support system, a client's attitudes towards mental health are shaped by his social circle and community, problems faced by rural and urban clients are different, children are quite dependent on their friends and parents regarding mental health, people tend to mimic popular attitudes about mental health. Finally, the counsellor must avoid directly confronting these beliefs as clients would be resistant to such attempts.

Stage 2: In this stage, the natural units of meaning expressed by the participants in the interviews are examined. A brief summary of the data is present in Table 2.

Table 2: Revelatory Themes

Revelatory Themes Identified

Lack of support systems

Shame and guilt

Isolation

Ostracism

Fear of diagnosis and medication

Faith healers and rituals

Families as religious and cultural conduits

Joint and nuclear families.

Rural and urban origin 
Religious and cultural beliefs.

Class and education

Age

Language

Counsellor as gurus

Media diagnosis

Institutions influence

Stage 3: In this stage, the central themes present in the previous stage are examined in light of the research question to see what they reveal. These revelatory themes are:

Lack of support systems: clients lack social support systems, unable and unwilling to communicate emotionally. The counsellors were the only support and they had to re-establish relationships with better emotional security.

Shame and guilt: clients feel a strong sense of shame about their feelings and behaviour, fearing the consequences of the responses if they were more open.

Isolation: clients lack the resources to go against community expectations and perceive certain issues as inappropriate outside their families.

Ostracism: clients are affected by harsh language and societal perceptions therefore go to therapy discreetly. They are perceived as being insane or extremely disturbed.

Fear of diagnosis and medication: clients fear being diagnosed. They denytheir illness and prefer the counsellor to tell them they are fine. They seek reassurance, dislikingthe side effects and seriousness of medication. In addition, they lack a foreseeable insight and stop their medication soon.

Faith healers and rituals: recommended by peers, clients go to these first and come to counselling as a last resort or use counselling in addition to these. Some were directed to therapy by faith healers.

Families as religious and cultural conduits: families are highly dependant on religious and social norms. Clients wish to comply 84 
with their family expectations. The appropriateness of going outside the family was a major concern.

Joint and nuclear family: joint and nuclear family determined emotional support systems. Rural migrants would have to change how they sought emotional support, grow more dependent and struggle between traditional and urban lifestyles. Families were hostile to ideas such as family therapy viewing it as foreign.

Rural and urban: the origins differentiated clients in the amount of help available, with rural clients having better-established support systems, more flexibility, and experiencing conflict when they migrated to urban centres. Urban clients had better access, were rigid, had no language barriers but lacked social support.

Religious and cultural beliefs: these were ingrained early and strongly held. Clients would respond positively to the incorporation of religious ideas in the therapeutic process, feeling more comfortable with people of similar religious backgrounds.

Class and education: they determinedthe resources and awareness with thehigher economic class being more open. Education affectedthe support offered but people were uninformed about mental health and questionedcounsellors less qualified than them.

Age: children were less rigid and responded to facts and reason. Older clients were resistant to change and other perspectives. The younger population had identity issues, relationship issues, mediafriendly and insightful, open to counsellors with different backgrounds.

Language: a lot of nuance about cultural beliefs, religion and tradition was lost when translated. Clients who couldn't express their emotions were taught to be more expressive. Certain therapies like ABA, done mostly in English, limited its accessibility.

Counsellor as guru: there was a tendency to see the counsellor as an ideal person devoid of any flaws.

Media diagnosis: clients would develop ideas about mental health from movies, their friend's circles and news stories. Many were confused by what they saw and would mislabel conditions, being resistant to clarifications. 
Institution's influence: institution's influence played a big role in the awareness, expectations, time, and intervention. Many institutions expected counsellors to be of a certain age, have children, and show immediate results.

Stage 4: It was found that families joint or nuclear serve as differing conduits for religious and cultural beliefs, the socio-economic class, rural or urban origin affect how the client responds and holds these worldviews. Clients were pushed to faith healers by their communities, faced ostracism, experienced shame, guilt, isolation, difficulties with language, saw counsellors as authority figures, made sense of their experiences from media and grew more rigid in their beliefs with age. Counsellors had to familiarise themselves, addressing them in a sensitive, non-confrontational manner while having a limited capacity to address the social environment

\section{Discussion}

The data obtained presents a detailed assessment of the various cultural signifiers and worldviews that counsellors had to accommodate. While existing research, Carson et al. (2009), had identified family as a major factor it had not identified it as playing a central role in religiousandcultural attitudes. Families saw therapy as foreign or threatening.

Similar to Kakar and Kakaer (2007) and Arulmani (2007), religionwould play a role through faith healers, rituals and so on. Clients came to therapy when these had failed to bring about change or as a supplement to these traditions.

In keeping with the research by Pereira and Rekha (2007) lack of awareness and stigma about mental health and counselling were key challenges in the field of counselling in India.

As the respondents in this research pointed out, learning and developing an attitude of openness to these cultural influencers would go a long way in making therapy more relevant and effective. It can provide a useful, specific guide for those who wish to understand and incorporate these influences in any way which does not exist in most literature of this nature. It can provide a guide to the relevant influenceslike culture, religionand so on. 
Further research and attempts to indigenous Indian psychology could examine the themes identified in this research.

\section{Limitations}

The study used a mixed sample in therapies that counsellors used for their clients. Narrowing in on a therapeutic and demographic approach might reveal more verifiable data. Since this research is qualitative, it is not easily generalisable. The sample only included practitioners in Bangalore; a study of counsellors across India might offer a more comprehensive picture.

\section{Recommendations}

The themes detailed in this study could be further explored to develop comprehensive, nuanced, estimates of their nature and manifestations. Quantitative evaluations limited to particular demographics and factors like religion, language, class or age groups could be conducted. Future research could evaluate the background of the counsellor or their therapeutic approach in how it affects the incorporation of indigenous psychology.

\section{Conclusion}

In order to gain a complete understanding of indigenous worldviews and their influence on the therapeutic process, it is necessary to study all relevant aspects of culture that might prove to be significant factors in therapy. This must also account for the multiplicities of therapeutic approaches that may differ in the importance they accord to particular elements within a social system such as family or social wellbeing. The therapeutic process shall be affected by differing social backgrounds, regional differences, personal orientations and so on as demonstrated by the findings of this study. Since each therapeutic model and socioeconomic variation leads to differences, it is important to examine what effect they bear on therapy across different regions and groups. Knowledge of this influence will aid in the further refining and adaptation of mental health practices in the country. 


\section{References}

Gould, S. J. (1981). The mismeasure of man. New York: W. W. Norton \& Company.

Kao \& D. Sinha, H. S., \& Sinha, D. (1997). The journey to the East an introduction. Asian Perspectives on Psychology, 9-22.

Mishra, R. C. (2006). Indian perspectives on cognition. In U. Kim, K. S. Yang, \& K. K. Hwang, Indigenous and cultural psychology (pp. 263- 281). New York: Springer.

Shweder, R. A. (2008). The cultural psychology of suffering: The many meanings of health in Orissa, India. Ethos, 60-77.

Adair, J. G. (1999). Indigenization of psychology: The concept and its practical implications. Applied Psychology an International Review, 403418.

Allwood, C. M. (2002). Indigenized psychologies. Social Epistemology: A Journal of Knowledge, Culture and Policy, 349- 366.

Allwood, C. M. (2011). On the foundation of the indigenous psychologies. Social Epistemology, 3-14.

Arulmani, G. (2007). Counselling psychology in India: At the confluence of two traditions. Applied psychology: an international review, 56(1), 6982.

Bhola, P., Kumaria, S., \& Orlinsky, D. E. (2012). Looking within: selfperceived professional strengths and limitations of psychotherapists in India. Asia Pacific Journal of Counselling and Psychotherapy, 161-174.

Carson, D. K., Jain, S., \& Ramirez, S. (2009). Counselling and family therapy in India: evolving professions in a rapidly developing nation. International Journal of Advanced Counselling, 45-56.

Davidsen, A. S. (2013). Phenomenological approaches in psychology and health sciences. Qualitative Research in Psychology, 318-333.

Harvey, L. (2019, January 25). Guide to methodology. Retrieved February 13, 2019, from Quality Research International: http:// www.quality researchinternational.com/methodology/RRW4pt2pt1Unstructured.p hp

Kakar, S. (1982). Shamans, mystics and doctors: A psychological inquiry into India and its healing traditions. New Delhi: Oxford India Perennials.

Kakar, S., \& Kakar, K. (2007). The Indians: portrait of a people. New Delhi: Penguin Books India.

King, R., \& Brien, T. O. (2011, August). Transference and countertransference: Opportunities and risks as twotechnical constructs migrate beyond their psychoanalytic homeland. Psychotherapy in Australia, 12-17. 
Kumaria, S., Bhola, P., \& Orlinsky, D. E. (2017). Influences that count: professional development of psychotherapists and counsellors in India. Asia Pacific Journal of Counselling and Psychotherapy, 1-21.

Levitt, S. (2017). Ethical issues in qualitative research. Newbury Park: SAGE publications.

Nandy. (2004). Towards an alternative politics of psychology. New Delhi, Delhi, India: Oxford University Press.

Pereira, M., \& Rekha, S. (2007). Problems, difficulties and challenges faced by counsellors. The International Journal of Indian Psychology, 65- 72.

Plucker, J. (1997, February). The Cyril Burt affair. Retrieved February, 2019, from Human Intelligence: http://www.indiana.edu/ intell/ burtaffair.shtml

Sinha, J. (1944). Indian psychology. Delhi: Jainendra Press.

Smith, D. W. (2018, June 21). Phenomenology. Retrieved February 13, 2019, from Stanford Encyclopedia of Philosophy: https: // plato.stanford.edu/cgi-bin/ encyclopedia/ archinfo.cgi? entry= phenomenology

Sriram, S., \& Duggal, C. (2015). The family courts act in India: perspectives from marriage counsellors. Indian Journal of Socio-Legal Studies, 96-103.

Teo, T. (2013). Backlash against American psychology: An indigenous reconstruction of the history of German critical psychology. History of Psychology, 1-18.

Whiting, L. (2002). Analysis of phenomenological data: personal reflections on Giorgi's method. Nurse Researcher, 9(2), 60-74. 